\title{
Percepção de enfermeiros sobre aspectos facilitadores e dificultadores de sua prática nos serviços de Saúde Mental
}

Perception of nurses about advantages and constraints of their practice in Mental Health services

Percepción de enfermeros sobre ventajas y limitaciones de su práctica en servicios de Salud Mental

\author{
Nathália Santos Silva', Elizabeth Esperidião", Ana Lúcia Queiroz Bezerra", \\ Ana Caroline Gonçalves Cavalcante", Adrielle Cristina Silva Souza", Kelly Kan Carvalho Silva" \\ 'Secretaria de Estado da Saúde de Goiás, Superintendência de Atenção Integral à Saúde, \\ Gerência de Saúde Mental. Goiânia-GO, Brasil. \\ " Universidade Federal de Goiás, Faculdade de Enfermagem. Goiânia-GO, Brasil.
}

Submissão: 30-08-2012 Aprovação: 16-09-2013

RESUMO

Trata-se de um estudo que teve como objetivo identificar os aspectos facilitadores e dificultadores das práticas dos enfermeiros nos serviços de Saúde Mental. Pesquisa descritiva de abordagem qualitativa, realizada em 22 serviços de Saúde Mental públicos do interior do Estado de Goiás. Foram sujeitos do estudo 21 enfermeiros. Utilizou-se o referencial de avaliação de Avedis Donabedian para discussão dos dados. Em relação aos aspectos facilitadores, foram citadas a disponibilidade de recursos físicos, mobiliário e materiais para realização das oficinas, qualificação profissional e relacionamento interpessoal com a equipe, coordenação e com os usuários. Entre os aspectos dificultadores predominaram também os relacionados à estrutura que os serviços oferecem. Ficou evidente que aspectos relacionados à estrutura e ao relacionamento interpessoal têm sido condicionantes para a efetividade das práticas dos enfermeiros em serviços de Saúde Mental no interior do estado de Goiás.

Descritores: Enfermagem Psiquiátrica; Enfermeiros; Serviços de Saúde Mental; Saúde Mental.

\section{ABSTRACT}

The study aimed to identify the advantages and constraints in the practices of nurses in Mental Health services. A qualitative descriptive study, was conducted in 22 public mental health services in the state of Goiás, involving 21 nurses as subjects. The evaluation referential of Avedis Donabedian was used for data discussion. Regarding the facilitating aspects, it were cited the availability of physical resources, furniture and materials for the workshops, professional and interpersonal relationships with staff, and coordination with users. Among the constraining aspects also predominated the structure that is offered by the services. It was evident that the structure and interpersonal relations have been conditioning aspects related to the effectiveness of nurses' practice in mental health services within the state of Goiás.

Key words: Psychiatric Nursing; Nurse; Mental Health Services; Mental Health.

\section{RESUMEN}

Se trata de un estudio que tuvo como objetivo identificar las ventajas y limitaciones de las prácticas de enfermería en los servicios de salud mental. El estudio tuvo un enfoque cualitativo descriptivo, y fue realizado en 22 servicios públicos de salud mental en el estado de Goiás. Los sujetos del estudio fueron 21 enfermeras. Se utilizó la evaluación de referencia de Avedis Donabedian para la discusión de los datos. En cuanto a los aspectos facilitadores fueron citados la disponibilidad de recursos físicos, el mobiliario y materiales para los talleres, las competencias profesionales y las relaciones interpersonales con el personal, y con la coordinación y los usuarios. Entre los aspectos que dificultan ha predominado también se la estructura de los servicios que ofrecen. Fue evidente que los aspectos relacionados con la estructura y las relaciones interpersonales han sido obstáculos para la efectividad de la práctica de enfermería en los servicios de salud mental en el estado de Goiás

Palabras clave: Enfermería Psiquiátrica; Enfermeras; Servicios de Salud Mental; Salud Mental.

\section{AUTOR CORRESPONDENTEＥlizabeth Esperidião E-mail: betesper@gmail.com}




\section{INTRODUÇÃO}

Nas últimas décadas do século XX, o movimento da Reforma Psiquiátrica no Brasil intensificou críticas à forma violenta como os pacientes eram tratados nos asilos e hospícios, ocasionando as primeiras tentativas de humanização desses $\operatorname{espaços~}^{(1)}$. A Reforma pretendeu modificar o sistema de tratamento clínico da doença mental, eliminando gradualmente a internação como forma de exclusão social e com o objetivo de integrar o sujeito que sofre de transtorno mental ao convívio familiar e social ${ }^{(2)}$.

Atualmente, o Brasil possui uma Política Nacional de Saúde Mental (PNSM) que abarca os princípios da Reforma Psiquiátrica e propõe consolidar o modelo de atenção que garante a livre circulação das pessoas com transtornos mentais e oferece cuidados com base nos recursos da comunidade. Com este enfoque, a rede de atenção ao portador de transtorno mental, deve ser formada por serviços comunitários articulados entre si, como os Centros de Atenção Psicossocial (CAPS), Residências Terapêuticas, Hospitais Gerais, Centros de Convivência, Hospitais-dia, Núcleos de Atenção Psicossocial (NASF), além da inclusão de beneficiários no Programa de Volta para Casa ${ }^{(3)}$.

Para compreender a singularidade dos indivíduos, num contexto social e coletivo, os serviços de atenção em Saúde Mental devem contar com equipe ampla e multidisciplinar, na qual o enfermeiro faz parte devendo, portanto implementar ações terapêuticas conforme orientações da PNSM.

O contexto atual do trabalho da enfermagem em Saúde Mental caracteriza-se pela transição entre uma prática de cuidado hospitalar que visava a contenção do comportamento dos doentes mentais e a incorporação de princípios novos e desconhecidos, na busca de adequação de práticas interdisciplinares ${ }^{(4)}$.

Com o surgimento dos serviços abertos de saúde mental, foi necessário reorganizar os processos de trabalho e, consequentemente o projeto terapêutico institucional, em que os profissionais da equipe multidisciplinar participam. Diante de muitas mudanças, coube também ao enfermeiro assumir novas funções com postura terapêutica, na perspectiva humanista e com autonomia profissional(5).

Com a mudança paradigmática da atenção à saúde mental, suas funções estão focadas na promoção da saúde mental, na prevenção da doença mental, na ajuda ao indivíduo a enfrentar as pressões decorrentes dos transtornos mentais e na capacidade de assistir ao paciente, sua família e comunidade, ajudando-os a encontrarem o verdadeiro sentido da enfermidade mental ${ }^{(6)}$.

Neste cenário, a prática atual da Enfermagem Psiquiátrica fundamenta-se em vários preceitos, incluindo a reabilitação psicossocial a que deve nortear suas ações junto aos usuários e familiares dos serviços de Saúde Mental. Há várias atividades terapêuticas desejáveis que os enfermeiros desenvolvessem nestes serviços, como relação de ajuda, atendimentos grupais e o relacionamento interpessoal, embora nem sempre sejam constantes e sistematizadas, mantendo suas ações de caráter assistencial-tecnicista contrariando o que é preconizado pela valorização das potencialidades e recuperação da autonomia do indivíduo em sofrimento psíquico, mediante uma abordagem compreensiva e individualizada ${ }^{(6-7)}$.

Para tanto, o enfermeiro deve usar a percepção e a observação, formular interpretações válidas, delinear seu campo de ação com tomada de decisões, planejar a assistência, avaliando as condutas e o desenvolvimento do processo. Essas ações fazem parte do cuidado de enfermagem, devendo direcionar o relacionamento interpessoal e terapêutico ${ }^{(6)}$.

Essa realidade vem requerer das instituições formadoras, a reconstrução dos seus Projetos Pedagógicos de Curso, no que se refere à atenção à saúde mental, buscando a reorientação do processo de formação voltado ao desenvolvimento de competências, para o exercício de práticas e saberes capazes de dar respostas aos princípios propostos pela Reforma Psiquiátrica(8).

Entretanto, os enfermeiros têm apresentado dificuldades em atender a PNSM, na medida em que não conseguem definir o objeto de trabalho de acordo com o paradigma da Reforma Psiquiátrica ${ }^{(1-2,4-5)}$. Na equipe de saúde mental, que define como objeto de intervenção o sujeito-cidadão em suas necessidades psicossociais, o enfermeiro não se define como sujeito-trabalhador ${ }^{(4)}$.

Tendo em vista que as transformações do modelo assistencial no campo da saúde mental mudaram a dinâmica no desempenho dos profissionais de Enfermagem, cabe questionar quais os fatores que influenciam positiva ou negativamente a prática destes profissionais nos serviços substitutivos especializados da área.

Com o intuito de aprofundar as reflexões decorrentes diante da problemática exposta, o objetivo do estudo foi identificar os aspectos facilitadores e dificultadores nas práticas dos enfermeiros nos serviços de Saúde Mental públicos do interior do Estado de Goiás.

\section{METODOLOGIA}

Pesquisa descritiva de abordagem qualitativa desenvolvida em todos os serviços públicos de Saúde Mental, 22 CAPS, do interior do estado de Goiás, habilitados até dezembro de 2010. Participaram os 21 enfermeiros que trabalhavam nestes serviços há mais de três meses. As perdas foram representadas por aqueles em afastamento oficial, como férias e licença.

A coleta de dados foi realizada entre janeiro e maio de 2011. Utilizou-se um instrumento autoaplicável, composto por questões fechadas sobre a caracterização dos enfermeiros e por questões abertas relacionadas à identificação dos aspectos facilitadores e dificultadores que os profissionais encontram para desenvolver suas atividades nos serviços.

As respostas foram transcritas e submetidas à análise de conteúdo, conduzida pela pré-análise, pela exploração do material e pelo tratamento e interpretação dos resultados. Assim, inicialmente, por meio da leitura flutuante os dados foram organizados para, na sequência, serem selecionados e classificados. Neste procedimento, procurou-se identificar palavras e frases que tivessem maior frequência de citação e que pudessem ser agrupadas em aspectos facilitadores ou dificultadores da prática dos enfermeiros, assim como a relevância 
de conteúdo expressa pelos participantes permitindo, deste modo, considerar os conteúdos com baixa frequência ${ }^{(9)}$.

Por fim, na fase de interpretação dos resultados os aspectos facilitadores e dificultadores foram categorizados utilizando-se o referencial de avaliação de Avedis Donabedian, com base na tríade: Estrutura, Processo e Resultados. Os aspectos relacionados à estrutura referem-se aos recursos físicos, humanos (número e qualificação), materiais (como instalações e equipamentos), financeiros e organizacionais. Quando se trata de processo, objetiva-se analisar todos os aspectos ligados às relações e às atividades desenvolvidas pelos profissionais de saúde, na condução dos cuidados e na interação com os usuários ${ }^{(10)}$.

Todo o processo de categorização foi analisado e discutido fundamentando-se nos documentos oficiais do Ministério da Saúde e na literatura especializada indexada em bases de dados. Os relatos dos enfermeiros apresentados nos resultados foram codificados com a letra " $E$ " seguidos do número da ordem com que foram entrevistados.

A pesquisa seguiu as orientações da Resolução no 196/96 tendo sido aprovada em Comitê de Ética em Pesquisa sob protocolo $\mathrm{n}^{\circ} 303 / 10$.

\section{RESULTADOS E DISCUSSÃO}

A maioria dos enfermeiros $(85,7 \%)$ era do sexo feminino, com idade entre 24 e 44 anos, sendo que a maior parte deles estava na faixa etária de 20 e 34 anos. Tinham pouco tempo de conclusão do curso de graduação e pouca experiência na área de Saúde Mental. Quase a metade dos enfermeiros $(47,6 \%)$ possuía até 2 anos de formados, 42,9\% até 4 anos e apenas 9,5\% tinha mais de 5 anos de conclusão do curso de graduação. Grande parte desses profissionais (76,2\%) estavam atuando no serviço há menos de 2 anos.

Tabela 1 - Aspectos facilitadores identificados pelos enfermeiros nos serviços de Saúde Mental do interior do estado de Goiás, 2011.

\begin{tabular}{ll}
\hline Variáveis & Frequência \\
\hline
\end{tabular}

\section{Relacionados à Estrutura dos serviços}

Disponibilidade de recursos materiais e físicos 5 Qualificação dos profissionais da equipe 3

Proximidade do serviço com a residência do profissional

Acesso à internet

Autonomia para realização de atividades de enfermagem

Ter equipe multiprofissional

Apoio e respeito da coordenação

Total

\section{Relacionados ao Resultado}

Trabalho em equipe

Obtenção de experiência na área de SM

Afinidade pela área, iniciativa, e motivação própria Interesse e aceitação (participação, empenho) dos usuários Comprometimento dos profissionais da equipe
Os enfermeiros possuíam vínculos empregatícios precários, visto que $85,7 \%$ estavam inseridos nos CAPS por meio de contratos de trabalho temporários. A jornada de trabalho variou entre 30 e 40 horas semanal, sendo que 71,4\% trabaIhavam em regime de 40 horas semanais.

Com relação à formação profissional, 57,1\% dos enfermeiros afirmaram possuir curso de pós-graduação Lato Sensu (especialização), embora apenas quatro na área de Saúde Mental. Nenhum enfermeiro atuante nos serviços de Saúde Mental de Goiás possui formação Stricto sensu.

Os resultados foram distribuídos em duas categorias: Aspectos facilitadores e Aspectos dificultadores, sendo que este último aborda estrutura e resultado ${ }^{(10)}$. Vale dizer que os enfermeiros não relataram atividades relacionadas ao processo, de acordo com o referencial de Avedis Donabedian.

\section{Aspectos facilitadores}

Os aspectos citados, pelos enfermeiros, como facilitadores para o desenvolvimento das suas atividades nos CAPS encontram-se listados na Tabela 1.

Dentre a maioria dos aspectos que facilitam o trabalho dos enfermeiros, prevalecem os relacionados à estrutura do serviço em detrimento dos resultados, os quais incluem a disponibilidade de recursos materiais e físicos, além da qualificação da equipe.

Especificamente em relação aos recursos físicos e materiais destacou-se o espaço, ambiente para atendimento aos usuários, mobiliário (mesas novas, cadeiras, armários) e materiais para realização das oficinas.

Trabalhar próximo ao serviço foi considerado como facilidade, aspecto também evidenciado em outro estudo, em que, somente a proximidade do local de trabalho com a residência do profissional foi um determinante externo ao ambiente de trabalho como causa de satisfação dos trabalhadores de saúde mental ${ }^{(11)}$.

Alguns enfermeiros citaram a qualificação dos profissionais da equipe multiprofissional e a experiência de seus membros na área de Saúde Mental como elementos que favorecem seu exercício profissional.

Sabe-se que a prática assistencial na área de saúde mental é complexa, pois requer, além da habilidade técnica necessária, a habilidade em lidar com o comportamento humano e com as relações humanas voltadas para a ressocialização do usuário ${ }^{(2)}$. Nesta perspectiva, a qualificação profissional é uma condição estruturante para o trabalho em Saúde Mental, segundo a lógica psicossocial, em virtude da reorganização do modelo assistencial proposto pela PNSM.

Os enfermeiros sentiram-se capacitados para atuar na área e relacionaram a realização de cursos de pós-graduação, especialmente Lato Sensu, conforme ilustra o depoimento a seguir:

Sinto-me capacitado sim. Por acreditar que a pós-graduação a qual cursei teve um altíssimo rendimento, tanto pelos excelentes monitores, como pelas experiências compartilhadas pelos colegas de classe que já trabalhavam nos serviços de saúde mental. (E17) 
Houve várias menções quanto a iniciativas próprias na busca por cursos, treinamentos e atualizações, a fim de melhorar o desempenho profissional.

Porque além de ser uma área que atuo porque gosto, tenho sempre me preparado em cursos, capacitações e diversas atualizações na área. Porém, a saúde mental nos surpreende a cada dia e por mais "capacitada" que eu seja, o serviço nos mostra que sempre temos que aprender e capacitar um pouco mais. (E3)

Foi destacado pelos profissionais, um aspecto importante relacionado ao resultado que é a afinidade pela área, iniciativa e motivação própria, que podem influenciar positivamente o desempenho profissional. Em contrapartida, a área de Saúde Mental parece não ser atrativa para alguns enfermeiros e, muitas vezes, não é a área de escolha, mas permanece nela por falta de melhores opções ${ }^{(1)}$.

Entende-se que ação profissional no campo da saúde é multideterminada a fatores ligados à formação acadêmica do enfermeiro, às características pessoais do profissional e da própria sociedade ${ }^{(1)}$.

Acredita-se que a baixa procura dos enfermeiros por trabalhos em Saúde Mental está intrinsecamente relacionada ao preconceito e estigma que muitas vezes não são superados pela forma com que o conteúdo é exposto durante os cursos de graduação, a pouca aproximação com os serviços extra-hospitalares e com os desafios postos pelo modelo psicossocial. Há também a desvalorização das competências relacionais, da criatividade e da plasticidade ${ }^{(12)}$.

Por outro lado, todos os aspectos facilitadores, identificados no aspecto Resultado, estão pautados no relacionamento interpessoal com a equipe e com os usuários: trabalho em equipe, apoio e respeito da coordenação, interesse e aceitação, participação e empenho dos usuários.

Esses aspectos facilitadores corroboram com os resultados de outro estudo que avaliou a satisfação dos trabalhadores dos serviços de saúde mental, no qual emergiram determinantes considerados internos ao ambiente de trabalho, a saber: as relações estabelecidas com os usuários, referida por todos os entrevistados, o reconhecimento profissional e as relações com a equipe ${ }^{(11)}$.

Entretanto, as relações no ambiente de trabalho podem ser causa de satisfação ou insatisfação, dependendo de como são estabelecidas $^{(11)}$. O apoio dos coordenadores nos serviços foi destacado por muitos enfermeiros como facilitador das atividades que desenvolvem, destacando o importante papel da gestão.

A receptividade dos coordenadores no sentido de escutar e acolher as demandas dos profissionais revela que o bom relacionamento possibilita a integração de equipe multiprofissional, na perspectiva de construção coletiva do trabalho nos CAPS ${ }^{(11)}$.

Diante das possibilidades de organização dos processos de trabalho, emergem novas formas de relação entre os trabalhadores, inclusive a necessidade do trabalho interdisciplinar ${ }^{(14)}$.

A interdisciplinaridade reforça a tendência de superação do compartilhamento do saber, como um dispositivo que busca integrar conhecimentos e atitudes que possam compreender a loucura em sua complexidade. A união de esforços pode ajudar na transformação do atendimento e no combate aos vícios de um modelo tradicional que ainda reside em nosso meio ${ }^{(13)}$. Elevados níveis de satisfação no trabalho na instituição correlacionam-se às boas relações profissionais, além de gostar de trabalhar com os usuários dos serviços ${ }^{(14)}$.

Entretanto, o trabalho interdisciplinar carece de constante reflexão. Não significa que todos podem fazer o trabalho de todos, porque há competências específicas de cada profissão. Certamente, a área da saúde mental passa por um período de reconstrução de práticas e saberes, tornando-se fundamental manter os profissionais engajados no compromisso coletivo. Espera-se que os profissionais de enfermagem trabalhem com a equipe no contexto da interdisciplinaridade, pois a inter-relação, a troca de experiências e conhecimentos favorece o cuidado junto ao usuário da rede de saúde mental(2).

\section{Aspectos dificultadores}

Os aspectos dificultadores foram mais identificados e, igualmente aos aspectos facilitadores, houve predominância de daqueles relacionados à Estrutura dos serviços.

Também foi possível identificar que alguns aspectos relacionados à Estrutura possuem uma característica dialética na medida em que foram identificados como facilitadores quando presentes e dificultadores quando ausentes. Entretanto, consideram-se os recursos materiais, humanos e físicos, qualificação profissional e acesso à internet como elementos fundamentais para o desenvolvimento das práticas dos enfermeiros.

Tal aspecto dialético, representado por aparente oposição, pode estar relacionado à desigualdade de contextos dos serviços situados em diversos municípios e, apesar de terem a mesma finalidade, têm estrutura e funcionamento diferentes, resultado confirmado com outro estudo(11).

A falta de recursos humanos, físicos e materiais como fator dificultador para o desenvolvimento das atividades nos serviços de saúde mental do interior do Estado de Goiás foi referida por mais da metade dos enfermeiros. Estudos apontam também que as principais causas de insatisfação dos trabalhadores relaciona-se aos aspectos inerentes à estrutura física e material, como inadequação do local, precariedade do ambiente e restrição de materiais para a realização das oficinas ${ }^{(1,11,14-15)}$.

Outro estudo realizado em Fortaleza corrobora com este resultado e mostra que $76 \%$ dos trabalhadores nos CAPS julgam que a estrutura física e a oferta de equipamentos/instrumentos para o trabalho não proporcionam condições satisfatórias para o desenvolvimento de suas ações ${ }^{(14)}$.

A preocupação com a estrutura física não tem origem somente na necessidade de conforto pessoal para a execução das atividades cotidianas, mas principalmente no desejo de melhor atender os usuários e de poder executar a contento as atividades programadas ${ }^{(11)}$, sendo, portanto um dos meios e instrumentos de produção de trabalho(10).

Três enfermeiros relataram também como entrave, a inexistência de transporte para realização das visitas domiciliares, impedindo a realização de buscas ativas, visitas para avaliar condições de tratamento oferecido no domicilio do usuário, transporte do usuário quando necessário, o que dificulta a 
Tabela 2 - Aspectos dificultadores identificados pelos enfermeiros nos serviços de Saúde Mental do interior do estado de Goiás, 2011.

\begin{tabular}{lc}
\multicolumn{1}{c}{ Variáveis } & Frequência \\
\hline Relacionados à Estrutura dos serviços & \\
Recursos materiais, humanos e físicos insuficientes & 11 \\
Transporte inexistente & 3 \\
Formação e capacitação profissional deficiente & 9 \\
Falta de acesso à internet & 1 \\
Vínculos empregatícios precários & 7 \\
Baixos salários & 1 \\
Ausência de trabalho interdisciplinar & 3 \\
Falta de apoio da coordenação & 1 \\
& 37 \\
Relacionados ao Resultado & Total \\
Centralidade no fazer médico & 2 \\
Falta de identificação do papel da enfermagem pela equipe multidisciplinar & 6 \\
Falta identificação do papel da enfermagem pelo próprio enfermeiro & 3 \\
Inexistência do trabalho em rede & 1 \\
Coordenação com perfil político & 1 \\
$\quad$ Total & 13 \\
\hline
\end{tabular}

adotar medidas que fortaleçam as condições de trabalho dos profissionais $^{(11,13)}$. Da mesma forma, os profissionais também devem reivindicar melhorias $^{(14)}$.

As deficiências podem ser superadas por meio da democratização das relações de trabalho, do resgate da participação dos profissionais na gestão do serviço e na assistência aos usuários. Para tanto, identificar o grau de comprometimento dos gestores dos serviços de saúde em viabilizar esse processo de democratização é fundamental ${ }^{(13-15)}$.

O papel do coordenador nos serviços de saúde mental no interior do Estado de Goiás foi um aspecto facilitador no sentido de apoiar os enfermeiros e direcionar o trabalho de toda a equipe. No entanto, o cargo de coordenador foi observado como negativo quando ele possui apenas característica política e não técnica. Entre os entrevistados esta situação não foi citada

assistência com qualidade. Houve relatos da utilização de veículo próprio do profissional para tal finalidade.

A disponibilidade de veículo no serviço para as visitas domiciliares é necessária, uma vez que, o tratamento tem como meta avaliar e auxiliar o portador de doença mental e sua família no contexto em que se encontra, além de facilitar a busca ativa de usuários que ausentes ou desistentes do tratamento. A falta de transporte contribui para o desconhecimento do território pela equipe e, portanto, para a ineficiência do tratamento.

Alguns sujeitos também citaram, como dificuldade, a falta de acesso à internet para realização de pesquisas. Sua utilização é um significativo meio por facilitar o acesso a informações e a processos de educação à distância. Fazer uso desta ferramenta auxilia programas de qualificação, o que permite a agilidade nos processos comunicacionais necessários à educação permanente ${ }^{(16)}$.

Vale dizer que no cenário de estudo, há muitos profissionais em regime de contrato de trabalho temporário/comissionado o que interfere no vínculo profissional e consequentemente na inserção em projetos assistenciais nos serviços. $\mathrm{O}$ fato de a maioria dos enfermeiros serem funcionários contratados enfraquece suas relações com a instituição. $\mathrm{O}$ vínculo frágil não garante a continuidade do trabalho e da assistência, como seria desejado ${ }^{(13-14,16)}$.

Outro fator desfavorável citado pelos participantes que precariza a realização do trabalho na enfermagem é o baixo salário dos enfermeiros. Este resultado foi igualmente apontado em outra localidade brasileira indicando consequências adversas, nas atividades desenvolvidas nos CAPS $^{(15)}$.

Diante deste panorama, fica evidente a necessidade dos gestores e coordenadores dos serviços de Saúde Mental em com frequência, embora mereça especial atenção pela complexidade da assistência da área de Saúde Mental que vem exigindo cada vez mais a mudança do modelo assistencial. Torna-se importante que os coordenadores dos serviços tenham clareza do seu papel a ser desempenhado nos serviços além das propostas da PNSM. Esta condição deve influenciar diretamente nas atividades dos enfermeiros.

O trabalho de enfermagem é marcado historicamente por subordinação ao modelo médico disciplinador e pelo desenvolvimento de práticas coadjuvantes. Entretanto, o enfermeiro terá mais condições de desenvolver práticas que visem o resgate da cidadania das pessoas em sofrimento mental se estiver consciente da sua condição social e pessoal e ao se perceber como corresponsável pelo trabalho com a coletividade ${ }^{(4)}$.

Outro resultado relevante corresponde à autonomia dada ao psiquiatra. Este fato reforça que muitos desses profissionais não se adequaram ao novo modelo de assistência em saúde mental que preconiza o trabalho multiprofissional e interdisciplinar.

A autonomia e centralidade nas consultas médicas estão relacionadas à deficiência de formação e capacitação dos enfermeiros. Na falta de um fazer definido o enfermeiro executa o que lhe parece correto ou o que se aproxima mais das ações que já está acostumado a realizar. Também o que poderia estar conduzindo a este posicionamento, seria o modelo de atenção à saúde ao qual o enfermeiro se insere. Trata-se de um modelo centrado na consulta médica, na psicofarmacologia e nos cuidados com o corpo, em virtude do agir terapêutico ${ }^{(17)}$.

A falta de capacitação na área de Saúde Mental, bem como a ausência de educação continuada foi considerada como aspectos dificultadores para o melhor desempenho das atividades profissionais dos enfermeiros. Talvez, por esta razão 
aparece entre as respostas de alguns participantes a não identificação de seu papel profissional nos serviços especializados.

"Eu não fui capacitada quando cheguei aqui. Cheguei e pronto! Fui trabalhar sem saber ao certo o que estava fazendo. Sinto falta disso".(E12)

Atualmente, verifica-se que, apesar da inserção do enfermeiro na equipe multiprofissional dos serviços de saúde mental, há ainda desvalorização das atividades específicas relativas à assistência ${ }^{(2)}$, a falta de reconhecimento pelos demais membros da equipe ${ }^{(1)}$. Percebe-se que, nos projetos institucionais, o enfermeiro está presente na equipe em função apenas de exigências legais e não como um profissional importante na assistência à pessoa em sofrimento psíquico ${ }^{(17)}$.

Diante disto, muitos enfermeiros não se sentem capacitados para atuar nos serviços de saúde mental e relaciona isto à deficiência do ensino durante a graduação à falta de educação continuada.

"Não me sinto capacitada, visto que não possuo nenhuma qualificação em saúde mental e creio que somente a graduação não oferece o suporte necessário para lidar adequadamente com o transtorno mental".(2)

"Não me sinto capacitada em totalidade, pois falta maior conhecimento acerca de procedimentos e condutas em saúde mental".(E11)

Ficou evidente que os profissionais necessitam de capacitação para desenvolver procedimentos específicos da área. Muitas vezes o enfermeiro não consegue estabelecer sua prática diferenciada da tradicional prática em psiquiatria indo das funções clássicas de supervisão, administração de medicamento, alimentação e higiene ${ }^{(2,7,18)}$.

O exercício da enfermagem, principalmente nos serviços de atenção psicossocial, deve se constituir pela responsabilidade na acolhida do usuário, estabelecendo vínculos afetivos, de confiança, de escuta e de relações interpessoais entre usuários e familiares. Torna-se imprescindível, portanto, que as universidades e os cursos de capacitação considerem a abordagem centrada no cliente e suas necessidades, na perspectiva da reabilitação e reinserção e social.

Há um distanciamento entre a teoria e prática desenvolvida nos serviços, o que pode ter influenciado no conhecimento do egresso que possui um domínio teórico limitado e centrado no modelo tradicional e segregante da psiquiatria. Esta realidade dificulta a percepção e a capacitação dos profissionais para as práticas atuais na assistência à Saúde Mental, com base na $\mathrm{PNSM}^{(2)}$. Salienta-se ainda que, grande parte dos cursos de graduação em Enfermagem está em municípios que não possuem CAPS, o que também dificulta a orientação psicossocial seja implementada pelos seus egressos.

Muitos autores têm enfatizado que para atuar nesta área, o enfermeiro deve ter preparo suficiente e de qualidade, com práticas que não se restrinjam ao hospital psiquiátrico, mas considerando o modelo psicossocial de atenção à saúde com enfoque na comunidade ${ }^{(1,16,18-19)}$. Sua inserção, enquanto discente, em serviços extra-hospitalares possibilita que vivencie e desenvolva competências que contemple o modelo psicossocial $^{(15,20)}$.

Apesar de não ter tido contato com serviços de saúde mental que não fosse o hospital psiquiátrico, enquanto acadêmica, estou sempre procurando aperfeiçoar meus conhecimentos. Senti falta desta prática quando entrei aqui no CAPS.(E7)

O curso de graduação deve possibilitar o pensamento teórico, permitindo a compreensão das novas demandas na formação de enfermeiros que apresentem uma identidade profissional ${ }^{(1-2,16-17,20)}$. No entanto, a realidade aponta como um dos aspectos dificultadores, a falta de preparo profissional especializado ${ }^{(1-2)}$

É fundamental direcionar a formação acadêmica envolvendo os enfermeiros e a equipe multiprofissional, atuantes nos serviços, como parte do processo educativo. A educação permanente é necessária e desejada pela equipe de enfermagem de saúde mental e exige além de programas educacionais baseados em definições de competências específicas, processos educativos mais amplos e problematizadores que visem o desenvolvimento de conhecimentos de caráter interdisciplinar ${ }^{(12,16)}$. Deve auxiliar no conhecimento de estratégias inovadoras de cuidar, favorecer o intercâmbio de experiências e a aliança entre os serviços de saúde e as instituições acadêmicas ${ }^{(16)}$.

A prática assistencial no campo da saúde mental é complexa, pois requer, além da habilidade técnica, destreza para lidar com as relações humanas e ressocialização do portador de transtorno mental ou em sofrimento psíquico.

A inexistência do trabalho em rede e a dificuldade na referência e contra-referência também apareceram como fator dificultador para o trabalho dos enfermeiros. A articulação da rede de serviços de saúde permite que os profissionais encaminhem os usuários a outros dispositivos de saúde, sejam eles na atenção básica ou nos demais serviços do território. No entanto, é possível verificar que os profissionais dos outros serviços de saúde dificultam a inserção daqueles referenciados pelo CAPS, possivelmente pelo estigma social que ainda existe com relação a essas pessoas, uma vez que a visão dos hospícios como proposta de tratamento para os doentes mentais não foi definitivamente descartada.

Espera-se que os CAPS sejam as unidades de saúde responsáveis pela porta de entrada dos usuários na rede especializada. Se esses serviços não tiverem os recursos necessários para a resolução do problema, deve-se encaminhar o usuário para a referência, que se constitui em outro serviço considerado de maior complexidade. Trata-se de estabelecer, portanto, a circulação controlada, para que os problemas sejam resolvidos com eficácia e ao menor custo possível ${ }^{(21)}$.

\section{CONSIDERAÇÕES FINAIS}

A assistência em enfermagem nos serviços de Saúde Mental, especialmente nos CAPS, pode ser influenciada por 
diversos fatores relacionados à disponibilidade de recursos, fatores inerentes aos profissionais, fatores relacionados à experiência na área, bem como a dinâmica do profissional com os demais membros da equipe de saúde.

Diante os resultados desta pesquisa, foi possível perceber que alguns enfermeiros consideraram que os fatores facilitadores colaboram para que os profissionais desenvolvam seu trabalho com interesse e motivação.

O estudo também contribuiu para o conhecimento das lacunas e deficiências que ainda existem nos CAPS, além de despertar para a necessidade de garantir meios/recursos para que os profissionais possam desenvolver ações fundamentadas na perspectiva humanista que prevê abordagem compreensiva e individualizadas.

É inegável a necessidade de adequação da Estrutura dos serviços no interior do estado de Goiás quanto aos espaços dos ambientes e disponibilidade de materiais. A ausência destes fatores tem dificultado o desenvolvimento das atividades no CAPS, prejudicado o tratamento dos usuários quanto a realização de oficinas terapêuticas sem espaço e material suficiente e acolhimento sem garantia de privacidade dos usuários e familiares.

Outra questão relacionada à Estrutura em destaque refere-se à falta de qualificação dos enfermeiros, cuja condição não favorece a visibilidade do profissional, gerando sensação de insegurança perante a equipe multiprofissional. Foi possível perceber, de forma antes empírica, alguns fatores que foram atribuídos à formação acadêmica, como a indefinição de papéis, a crise de identidade do profissional enfermeiro, a dificuldade em trabalhar com os usuários nos serviços de saúde mental e com a equipe multiprofissional. Muitas vezes a concepção do modelo psicossocial o qual concebe práticas que contrapõem as práticas asilares e médico-centradas, deixa de ser aprofundadas na capacitação profissional, comprometendo o atendimento do usuário do serviço de modo integral e humanizado.

É premente que as instituições formadoras adequem o ensino da Enfermagem Psiquiátrica e de Saúde mental ao modelo proposto pela PNSM, inserindo estágios e aulas práticas nos serviços que compõem a rede especializada em Saúde Mental, em especial os CAPS.

Como forma de agregar conhecimentos, a educação permanente é importante, visto a necessidade de formar e manter os recursos humanos qualificados e cientes das diretrizes da Política Nacional de Saúde Mental.

Por fim, os processos avaliativos têm que ser reforçados com o intuito de conhecer a situação dos serviços, suas fragilidades e potencialidades e, desta forma, discutir ações para a melhoria da formação e práticas dos enfermeiros no contexto da saúde mental.

\section{REFERÊNCIAS}

1. Dias CB, Aranha e Silva AL. O perfil e a ação profissional da(o) enfermeira(o) no Centro de Atenção Psicossocial. Rev Esc Enferm USP. [periódico na internet]. 2010 [acesso em 02 mar 2011];44(2):469-75. Disponível em: www. ee.usp.br/reeusp/

2. Calgaro A, Souza EN. Percepção do enfermeiro acerca da prática assistencial nos serviços públicos extra-hospitalares de saúde mental. Rev Gaúch Enferm. 2009;30(3):476-83.

3. Ministério da Saúde. Secretaria de Atenção à Saúde. DAPES. Coordenação Geral de Saúde Mental, Álcool e Outras Drogas Saúde Mental no SUS: as novas fronteiras da Reforma Psiquiátrica. Relatório de Gestão 2007/2010. Brasília, DF: Ministério da Saúde; 2011.

4. Oliveira AGB, Alessi NP. O trabalho de enfermagem em saúde mental: contradições e potencialidades atuais. Rev Latino-am Enferm. 2003;11(3):333-40.

5. Vilela SC, Moraes MC. A prática de enfermagem em serviços abertos de saúde mental. Rev Enferm UERJ. 2008;16(4):501-6.

6. Villela SC, Scatena MCM. A Enfermagem e o cuidar na área de Saúde Mental. Rev Bras Enferm. 2004 57(6):738-41.

7. Esperidião E, Cruz MFR, Silva GA. Perfil e atuação dos enfermeiros da rede especializada em saúde mental de Goiânia-Goiás. Rev Eletrônica Enferm [periódico na internet]. 2011;13(3):493-501. Disponível em: http://www. fen.ufg.br/revista/v13/n3/v13n3a15.htm

8. Fernandes JD, Sadigursky D, Silva RMO, Amorim AB,
Teixeira GAS, Araújo MCF. Ensino da enfermagem psiquiátrica/saúde mental: sua interface com a reforma psiquiátrica e diretrizes curriculares nacionais. Rev Esc Enferm USP. 2009;43(4):962-8.

9. Bardin L. Análise de Conteúdo. 4. ed. Lisboa: Edições 70; 2010.

10. Donabedian A. Quality assurance. Structure, process and outcome. Nurs Stand. 1992;7(11 Suppl QA):4-5.

11. Guimarães JMX, Jorge MSB, Assis MMA. (In)satisfação com o trabalho em saúde mental um estudo em Centros de Atenção Psicossocial. Ciênc Saúde Coletiva. 2011;16(4):2145-54.

12. Lucchese R. A enfermagem psiquiátrica e saúde mental: a necessária constituição de competências na formação e na prática do enfermeiro. Rev Eletrônica Enferm [periódico na internet]. 2007 [acesso em 30 mar 2010];9(3):883-5. Disponível em: http://www.fen.ufg.br/revista/v9/n3/v9n3a28.htm.

13. Pinho LB, Hernández AMB, Kantorski LP. Trabalhadores em saúde mental: contradições e desafios no contexto da reforma psiquiátrica. Esc Anna Nery Rev Enferm. 2010;14(2):260-7.

14. Jorge MSB, Guimarães JMX, Nogueira MEF, Moreira TMM, Morais APP. Gestão de recursos humanos nos Centros de Atenção Psicossocial no contexto da política de desprecarização do trabalho no Sistema Único de Saúde. Texto \& Contexto Enferm. 2007; 16(3):417-25.

15. Rézio LA, Oliveira AGB. Equipes e condições de trabalho nos centros de atenção psicossocial em Mato Grosso. Esc 
Anna Nery Rev Enferm. 2010;14(2):346-54.

16. Tavares CMM. A educação permanente da equipe de enfermagem para o cuidado nos serviços de saúde mental. Texto \& Contexto Enferm. 2006;15(2):287-95.

17. Rodrigues RM, Schneider JF. A enfermagem na assistência ao indivíduo em sofrimento psíquico. Rev Latino-Am Enferm. 1999;7(3):33-40.

18. Oliveira FB, Silva KMD, Silva JCC. Percepção sobre a prática de enfermagem em Centros de Atenção Psicossocial. Rev Gaúch Enferm. 2009;30(4):692-9.
19. Kantorski LP, Mielke FB, Teixeira Júnior S. O trabalho do enfermeiro nos centros de atenção psicossocial. Trab Educ Saúde. 2008;6(1):87-105.

20. Soares RD, Villela JC, Borba LO, Brusamarello T, Maftum MA. O papel da equipe de enfermagem no centro de atenção psicossocial. Esc Anna Nery Rev Enferm 2011;15(1):110-5.

21. Zambenedetti G, Silva RAN. A noção de rede nas reformas sanitária e psiquiátrica no Brasil. Psicol Rev. 2008;14(1):131-50. 